\title{
The High-Grade Glial Component Of Pediatric Primary Anaplastic Ganglioglioma Characterized Astroblastoma-Like Pseudorosettes With BRAFV600E Mutation And Deletion Of CDKN2A/B, PTEN, And BMPR1A: A Case Report
}

\section{Duan Zejun}

Capital Medical University

\section{Yao Kun}

Capital Medical University

Feng Jing

School of Life Sciences Beijing Normal University

Qi Xueling ( $\nabla$ xszqxl169@mail.ccmu.edu.cn)

Capital Medical University

\section{Case Report}

Keywords: Ganglioglioma, astroblastoma-like pseudorosettes, anaplastic ganglioglioma, BRAF, CNKN2A/B

Posted Date: October 5th, 2021

DOl: https://doi.org/10.21203/rs.3.rs-934230/v1

License: (c) (1) This work is licensed under a Creative Commons Attribution 4.0 International License. Read Full License 


\section{Abstract}

Background: Ganglioglioma (GG) is a low-grade mixed neuronal-glial tumor which is the most common type of long-term epilepsy-associated tumors (LEATs). However, primary anaplastic ganglioglioma (AGG) which composes of malignant changes is rare. Here, we report a case of pediatric primary AGG which is consisted of low-grade/begnin GG and high-grade glioma that was characterized by astroblastoma-like pseudorosettes.

Case presentation: We describe a case of 4-year-old female who presented with medically refractory seizure for 14 months by a temporal mass. The patient underwent a gross total mass resection at the first surgery, and was only treated with antiepileptic therapy and followed by observation. After nine months, tumor recurrence was found. Followed by second operation, the patient was treated with chemotherapy (oral temozolomide and antiepileptic drugs) and local radiotherapy. At 58-month follow-up after the second operation, no epileptic seizures and tumor recurrence were found again. In the first sample, the tumor contained two different components. The major component presented the low-grade GG's features of neoplastic glial cells and dysplastic ganglion cells. The minor component was a heterogeneous highgrade glioma characterized astroblastic-like pseudorosettes clusters with increased mitotic figure (about 4-6 per 10 high-power fields). CD34 staining was negative. BRAFV600E was positive in both components. In the recurrent sample, the heterogeneous high-grade glioma became the major component. The fuorescence in situ hybridization (FISH) of MN1 break-apart probe and MYB-QKI fusions probe were negative. $B R A F^{V 600 E}$ mutation, and deletion of $C D K N 2 A / B, P T E N$ and BMPR1A were detected by targeted DNA sequencing.

Conclusion: This case extends the histomorphologic spectrum and enriched genetic features of primary AGG in childhood. The high-grade glioma charactered astroblastoma-like pseudorosettes may be an important cause of tumor recurrence in a short period of time. Tumor gross total surgical resection and adjuvant chemoradiotherapy were important to achive an event-free survival.

\section{Background}

Ganglioglioma (GG) is a low-grade mixed neuronal-glial tumor which preferentially occurs in temporal lobe of children and adolescents, and which is the most common type of long-term epilepsy-associated tumors (LEATs) ${ }^{[1,2]}$. GG is a well-differentiated, slow-growing giloneuronal neoplasm composed of dysplastic ganglion cells in combination with neoplastic glial cells. Anaplastic ganglioglioma (AGG) rarely represent $1-30 \%$ of $\mathrm{GGs}^{[3]}$ and categorized as grade 3 according to WHO classification, which composed of dysplastic ganglion cells and an anaplastic glial component with increased proliferative index, angiogenesis and necrosis. Here, we report a case of pediatric primary AGG which is consisted of low-grade/begnin GG and high-grade glioma that was characterized by astroblastoma-like pseudorosettes.

\section{Case Presentation}


A 4-year-old female presented with medically refractory seizure for 14 months. The preoperative magnetic resonance imaging (MRI) showed the mass in the temporal with slight hypointense on T1-wighted image (T1WI) (Fig. 1. A), hyperintense on T2-weighted image (T2WI) (Fig. 1. B) and on Flair sequence (Fig. 1. C). After the enhancement, the lesion was mildly enhanced (Fig. 1. D). The CT scan showed that there was obvious calcification within tumor (Fig. 1. E). The patient underwent a gross total mass resection in the right anterior and medial temporal that confirmed by postoperative CT (Fig. 1. F). Postoperatively, the patient was only treated with antiepileptic therapy and followed by observation. After nine months, tumor recurrence was found by MRI. Followed by second operation, the patient was treated with chemotherapy (oral temozolomide and antiepileptic drugs) and local radiotherapy. At 58-month follow-up after the second operation, no epileptic seizures and tumor recurrence were found. This study was approved by the ethical committee of Beijing Sanbo Brain Hospital.

Histologically, the lesion located in the cortex involving with the hippocampus and subarachnoid space. The tumor contained two different components (Fig. 2). The major component presented the GG's features of glial and neuronal cell elements combination. The glial cells with mild nuclear atypia resembled fibrillary astrocytoma. Occasional mitoses were observed in the glial component. The neuronal cells appeared the features of dysplastic ganglion cells, which included the vacuolar nucleus and obvious nucleoli. There were dystrophic calcification and prominent capillary network. The neoplastic glial cells had diffused immunoreactivity for GFAP, S-100, Nestin, OLIG2. Neuronal proteins markers, such as MAP2, synaptophysin (Syn), chromogranin-A (CgA), neurofilaments (NF) and NeuN, displayed scattered positivity in the dysplastic neurons. CD34 staining was negative in this case. BRAFV600E was positive in both glial and neuronal cells. The Ki-67 staining showed positivity only in the glial component as a ratio about $1-2 \%$. The minor component $(<5 \%)$ was a heterogeneous high-grade glioma characterized astroblastic-like pseudorosettes clusters, distributing among the major component. Astroblastic-like pseudorosettes were composed of elongated tumor cells containing abundant esosinophilic cytoplasm. Tumor cells distributed around the blood vessels in single-layered or pseudostratified form with a broad process. Mitoses were about 4-6 per 10 high-power fields. Astroblastic-like pseudorosettes presented diffused immunoreactivity for S-100, Nestin, Vimentin, and BRAFV600E, but were negative for GFAP, OLIG2, Syn, NF, NeuN, and CD34. EMA immunoreactivity showed a patchy pattern as dot-like perinuclear structures. The Ki-67 proliferation index was up to 15\%. Microvascular proliferation and necrosis were not observed. The heterogeneous high-grade glioma characterized astroblastic-like pseudorosettes became the major component at tumor recurrence (Fig. 3). However, the minor component (about 20\%) with mild nuclear atypia was similar to low-grade fibrillary astrocytoma, there was not obvious dysplastic neurons or ganglion cells. For both components in the recurrence tumor sample, immunohistochemical characteristics were also similar to the initial.

Targeted DNA sequencing was performed in formalin-fixed paraffin-embedded samples (425-cancerrelevant genes, Geneseeq Technology Inc.) (Supplementary Table 1). BRAF mutation (c.1799T > A, p.V600E) was identified with the mutant allele at a frequency of $30.84 \%$ (Fig. 4. A). The deletion copy number variations (CNV) of CDKN2A (copy number: 0.2984), CDKN2B (copy number: 0.3259), PTEN (copy number: 0.5346), and BMPR1A (copy number: 0.5229) were also be detected in this case (Fig. 4. B). 
Meanwhile, fluorescence in situ hybridization (FISH) was performed with MN1 break-apart probe and MYB-QKI fusions probe in the astroblastoma-like pseudorosettes. The results of FISH were all negative that different from astroblastoma ${ }^{[4]}$ and angiocentric gliomas ${ }^{[5]}$.

The final pathological diagnosis was primary AGG consisted of low-grade/begnin GG and a heterogeneous high-grade glioma characterized astroblastic-like pseudorosettes. BRAF ${ }^{\mathrm{V} 600 \mathrm{E}}$ mutation, CDKN2A homozygous deletion, and deletion of PTEN and BMPR1A were observed. Although CD34 is negative which is consistently expressed in GGs $(86.7 \%)^{[6]}$, we still diagnosed the major component as low-grade GG since it fulfilled other GG criteria established by : 1) children with medically refractory seizure and tumor located in the temporal; 2) morphological features consisted of neoplastic glial cells and dysplastic ganglion cells; 3 ) positive staining of BRAFV600E in both glial and dysplastic ganglion cells. About the heterogeneous high-grade glioma characterized astroblastic-like pseudorosettes, it was diagnosed as anaplastic glial component of AGG.

\section{Discussion And Conclusions}

Primary AGG is rare. Here, we report a case of pediatric primary AGG which is consisted of low-grade GG and high-grade glioma that was characterized by astroblastoma-like pseudorosettes. The glial component of most reported AGGs presented diffusely infiltative glioma ${ }^{[7]}$. In this case, the anaplastic glial element was characterized with astroblastoma-like pseudorosettes that were never reported in AGG before.

Moreover, BRAF ${ }^{\mathrm{V} 600 \mathrm{E}}$ mutation, $\mathrm{CDKN} 2 \mathrm{~A} / \mathrm{B}$ homozygous deletion, deletion of PTEN and BMPR1A were detected by targeted $D N A$ sequencing in this case. BRAF ${ }^{\mathrm{V} 600 \mathrm{E}}$ mutation is the most common genetic alteration in the $\mathrm{GG}$, occurring in $40-66 \%[8,9]$. BRAF ${ }^{\mathrm{V} 600 \mathrm{E}}$ mutation was frequently associated with additional alterations, most commonly deletion of CDKN2A/B, other alteration including NF1, FGFR1, KRAS, H3F3A, and so on ${ }^{[10]}$. The deletion of CDKN2A/B was present in some brian tumor types, including pediatric glioma and IDH-mutant astrocytoma ${ }^{[11]}$, which was also reported in AGG by array CGH analysis ${ }^{[12]}$. PTEN and BMPR1A are tumor suppressor genes which are located in close proximity on chromosome 10. Mutations or deletions of PTEN are frequent events in GBM $(36 \%)^{[13]}$ and correlate with poor prognosis ${ }^{[14]}$. And loss of PTEN is a late and critical event in GBM progression. PTEN and BMPR1A deletion dysregulates both the BMP-SMAD and PI3K-AKT-mTOR signaling pathways, promoting cell proliferation and survival in oncogenic astrocytes ${ }^{[15,16]}$. We suppose BRAF ${ }^{\mathrm{V} 600 \mathrm{E}}$ mutations was a driver alteration, CDKN2A/B homozygous deletion, deletion of BMPR1A, and PTEN increased the malignant risk and evolution.

In general, GG have a good prognosis after surgical resection, but AGG had a poor survival, the 5-year overall survival rate was about $24.9-63 \%^{[3,17-19]}$. Some study showed that the prognosis of pediatric patients with AGG is good, especially for those who undergo gross total tumor resection, and the mean 5year OS estimation and standard error was $88 \pm 12 \%[20]$. In view of recent studies, some studies reported 
that adjuvant radiotherapy did not influence overall survival (OS) of AGG, but surgery is an important predictor of $0 S^{[17]}$. Others thought optimal treatment was maximal safe surgical resection followed by postoperative radiotherapy and chemotherapy for AGG or GG composite anaplastic entity $[3,17,18]$. In our experience of this case, tumor gross total surgical resection was important and adjuvant chemoradiotherapy is also needed. The high-grade glioma charactered astroblastoma-like pseudorosettes in AGG may be an important cause of tumor recurrence in a short period of time. Surgery adjuvant local radiotherapy and chemotherapy could better help control the tumor recurrence.

In summary, there are three particular reasons to present this case. First, this case extends the histomorphologic spectrum of primary AGG, in which the high-grade glial component charaterized astroblastoma-like pseudorosettes. Second, it enriched the genetic features of primary AGG in childhood. $\mathrm{BRAF}^{\mathrm{V} 600 \mathrm{E}}$ mutation, CDKN2A/B homozygous deletion, deletion of PTEN and BMPR1A were observed in this AGG case, that was never reported before by our knowlege. Third, the high-grade glioma palyed an important role in tumor recurrence. Tumor gross total surgical resection and adjuvant chemoradiotherapy were important to achive an event-free survival.

\section{Abbreviations}

GG:Ganglioglioma;

LEATs: long-term epilepsy-associated tumors;

AGG:anaplastic ganglioglioma;

FISH:fuorescence in situ hybridization;

MRI:magnetic resonance imaging;

T1WI:T1-wighted image;

T2Wl:T2-weighted image;

Syn:synaptophysin;

CgA:chromogranin-A;

NF:neurofilaments;

OS:overall survival

\section{Declarations}

Acknowledgment: Thank individuals who contributed to the study or manuscript preparation but who do not fulfill all the criteria of authorship. 
Authors' contributions: Overall experimental design was conceived and supervised by Xueling Qi. Zejun Duan and Ke Xu contributed to the data analysis and final draft of the manuscript. Jing Feng helped to polish the article. All authors and approved the final manuscript.

Funding: No funding received.

Availability of data and metericals: The data supporting the findings of this study are available on request from the corresponding author.

Declarations: All authors state that the views expressed in the submitted article are his or her own and not an official position of the institution or funder.

Conflicts of interest/Competing interests : All authors declare that they have no conflicts of interest.

Patient perspective: This report got the consent from the patient's legal representatives.

\section{References}

1. Perry A, Giannini C, Scheithauer BW, et al. Composite pleomorphic xanthoastrocytoma and ganglioglioma: report of four cases and review of the literature. Am J Surg Pathol. 1997;21(7):76371.

2. Blumcke I, Spreafico R, Haaker G, et al. Histopathological Findings in Brain Tissue Obtained during Epilepsy Surgery. N Engl J Med. 2017;377(17):1648-56.

3. Terrier LM, Bauchet L, Rigau V, et al. Natural course and prognosis of anaplastic gangliogliomas: a multicenter retrospective study of 43 cases from the French Brain Tumor Database. Neuro Oncol. 2017;19(5):678-88.

4. Mhatre R, Sugur HS, Nandeesh BN, Chickabasaviah Y, Saini J, Santosh V. MN1 rearrangement in astroblastoma: study of eight cases and review of literature. Brain Tumor Pathol. 2019;36(3):11220.

5. Bandopadhayay $P$, Ramkissoon LA, Jain $P$, et al. MYB-QKI rearrangements in angiocentric glioma drive tumorigenicity through a tripartite mechanism. Nat Genet. 2016;48(3):273-82.

6. Wang Y, Wang L, Blümcke I, et al. Integrated genotype-phenotype analysis of long-term epilepsyassociated ganglioglioma. Brain Pathol. 2021: e13011.

7. Lisievici AC, Pasov D, Georgescu TA, et al. A novel histopathological grading system for ganglioglioma. J Med Life. 2021;14(2):170-5.

8. Prabowo AS, lyer AM, Veersema TJ, et al. BRAF V600E mutation is associated with mTOR signaling activation in glioneuronal tumors. Brain Pathol. 2014;24(1):52-66.

9. Martinoni M, Marucci G, de Biase D, et al. BRAF V600E mutation in neocortical posterior temporal epileptogenic gangliogliomas. J Clin Neurosci. 2015;22(8):1250-3. 
10. Ryall S, Zapotocky M, Fukuoka K, et al. Integrated Molecular and Clinical Analysis of 1,000 Pediatric Low-Grade Gliomas. Cancer Cell. 2020;37(4):569-83.e5.

11. Appay $R$, Dehais $C$, Maurage $C A$, et al. CDKN2A homozygous deletion is a strong adverse prognosis factor in diffuse malignant IDH-mutant gliomas. Neuro Oncol. 2019;21(12):1519-28.

12. Reifenberger G, Collins VP. Pathology and molecular genetics of astrocytic gliomas. J Mol Med (Berl). 2004;82(10):656-70.

13. Comprehensive genomic characterization. defines human glioblastoma genes and core pathways. Nature. 2008. 455(7216): 1061-8.

14. Srividya MR, Thota B, Shailaja BC, et al. Homozygous 10q23/PTEN deletion and its impact on outcome in glioblastoma: a prospective translational study on a uniformly treated cohort of adult patients. Neuropathology. 2011;31(4):376-83.

15. Taylor H, Yerlioglu D, Phen $\mathrm{C}$, et al. mTOR inhibitors reduce enteropathy, intestinal bleeding and colectomy rate in patients with juvenile polyposis of infancy with PTEN-BMPR1A deletion. Hum Mol Genet. 2021;30(14):1273-82.

16. Hover LD, Owens $\mathrm{P}$, Munden AL, et al. Bone morphogenetic protein signaling promotes tumorigenesis in a murine model of high-grade glioma. Neuro Oncol. 2016;18(7):928-38.

17. Selvanathan SK, Hammouche S, Salminen HJ, Jenkinson MD. Outcome and prognostic features in anaplastic ganglioglioma: analysis of cases from the SEER database. J Neurooncol. 2011;105(3):539-45.

18. Rosselló A, Plans G, Vidal-Sarró N, Fernández-Coello A, Gabarrós A. Ganglioglioma Progression to Combined Anaplastic Ganglioglioma and Anaplastic Pleomorphic Xanthoastrocytoma. Case Report and Literature Review. World Neurosurg. 2017;108:996. .e17-996.e25..

19. Lucas JT Jr, Huang AJ, Mott RT, Lesser GJ, Tatter SB, Chan MD. Anaplastic ganglioglioma: a report of three cases and review of the literature. J Neurooncol. 2015;123(1):171-7.

20. Karremann M, Pietsch T, Janssen G, Kramm CM, Wolff JE. Anaplastic ganglioglioma in children. J Neurooncol. 2009;92(2):157-63.

\section{Figures}



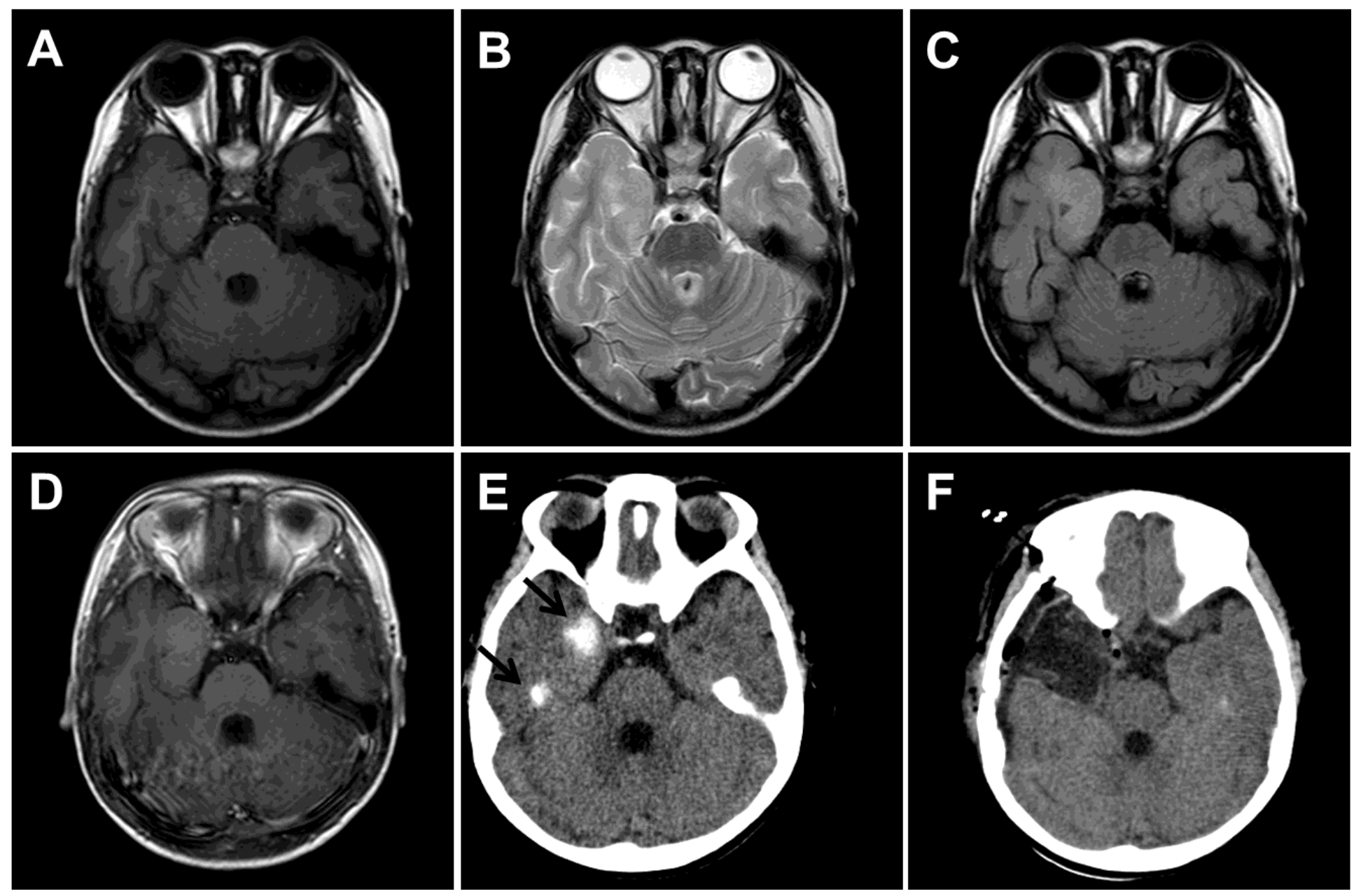

Figure 1

The first preoperative and postoperative radiographic imagings. MRI showed the mass with slight hypointense T1-weighted signal intensity (A) and hyperintense T2-weighted signal intensity (B), hyperintense FLAIR sequence intensity with unclear boundary (C). After the enhancement, the lesion was mildly enhanced (D). CT showed the right temporal lobe medial swelling with obvious calcification (black arrows) (E). Postoperative CT confirmed gross total mass resection (F). 


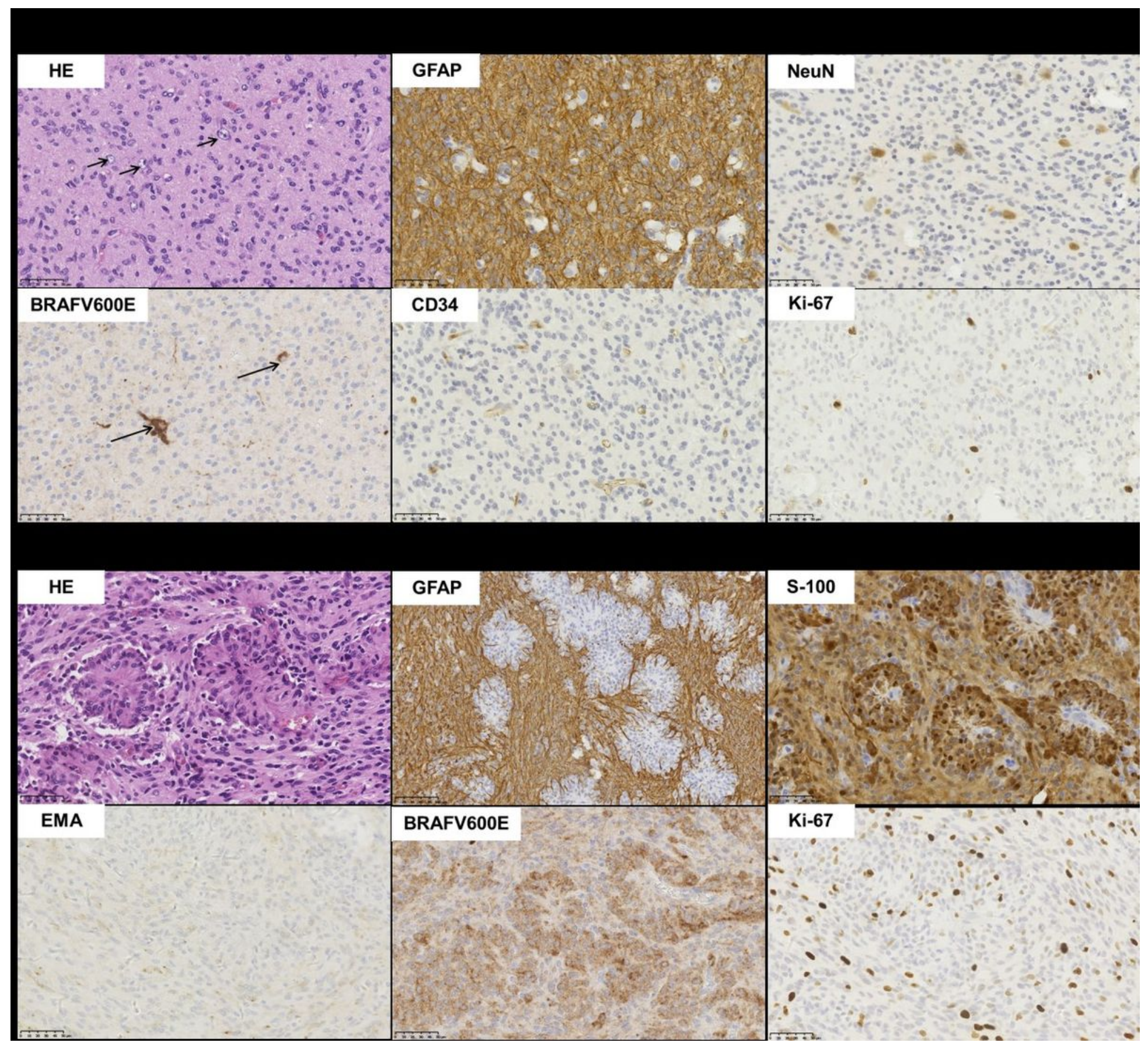

Figure 2

Histological features and immunohistochemical findings of the first surgery specimens (x400). A GG. Combination of glial and neuronal cell elements in GG (short arrows showed the dysplastic neurons). The neoplastic glial cells were diffuse immunoreactivity for GFAP. The dysplastic neurons were positive for NeuN. BRAFV600E was strong positive staining in scattered dysplastic neurons and weak in glial cells (long arrows showed the positive staining dysplastic neurons). CD34 was negative. Ki-67 proliferation index was about 1-2\%. B Astroblastoma-like pseudorosettes. Astroblastoma-like pseudorosettes were composed of single-layered or pseudostratified elongated tumor cells with a broad process and esosinophilic cytoplasm extending to the central blood vessel. GFAP was negative in pseudorosettes. S- 
100 has diffuse strong expression. EMA showed a patchy dot-like perinuclear structures. BRAFV600E was strong positive staining. Ki-67 proliferation index was up to $15 \%$.

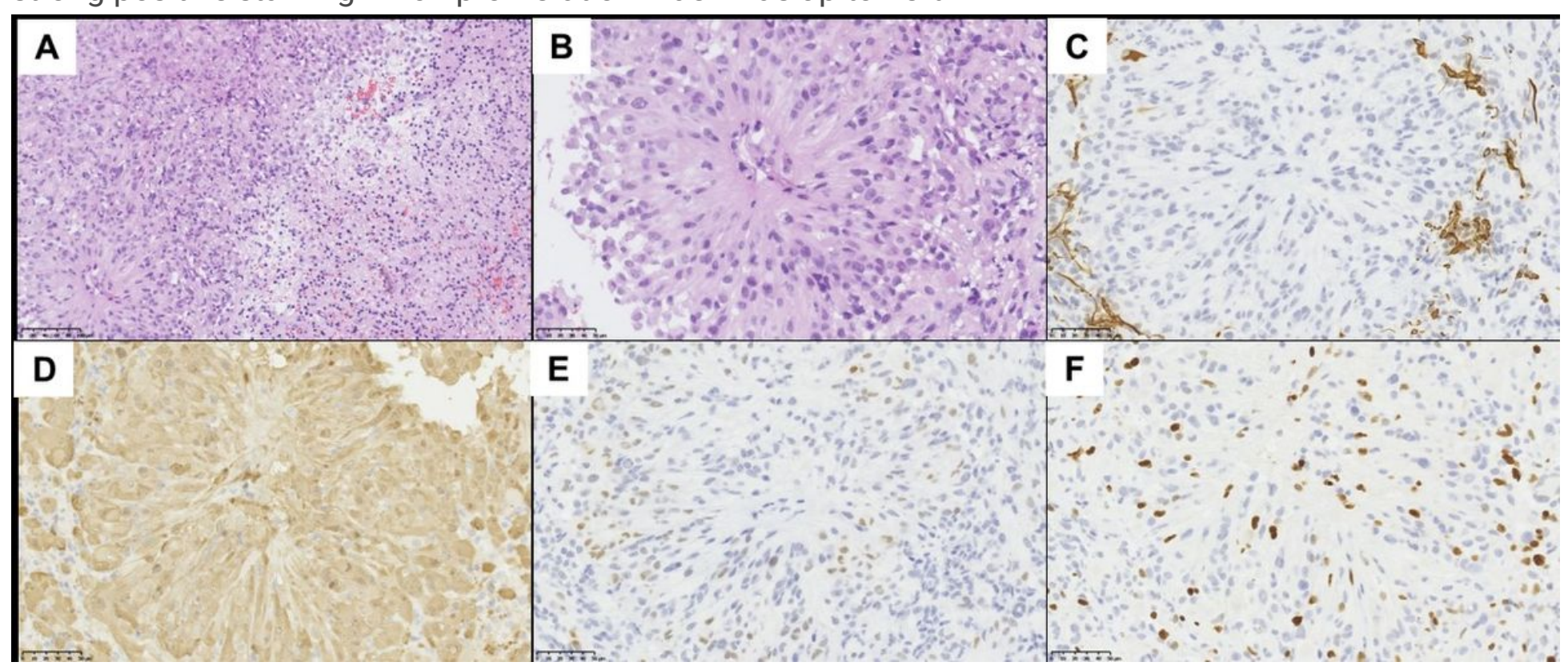

\section{Figure 3}

Histological features and immunohistochemical findings of tumor recurrence in two different components. The left is the astroblastoma-like pseudorosettes element, and the right is like low-grade glioma without obvious dysplastic neurons $(A, \times 200)$. Astroblastoma-like pseudorosette is composed of pseudostratified elongated tumor cells extending to the central blood vessel $(B, \times 400)$. GFAP was negative staining $(C, \times 400)$. S-100 was diffuse strong positve $(D, \times 400)$. OLIG2 was positve staining in some tumor cells $(E, \times 400)$. Ki-67 was about $15 \%(F, \times 400)$. 


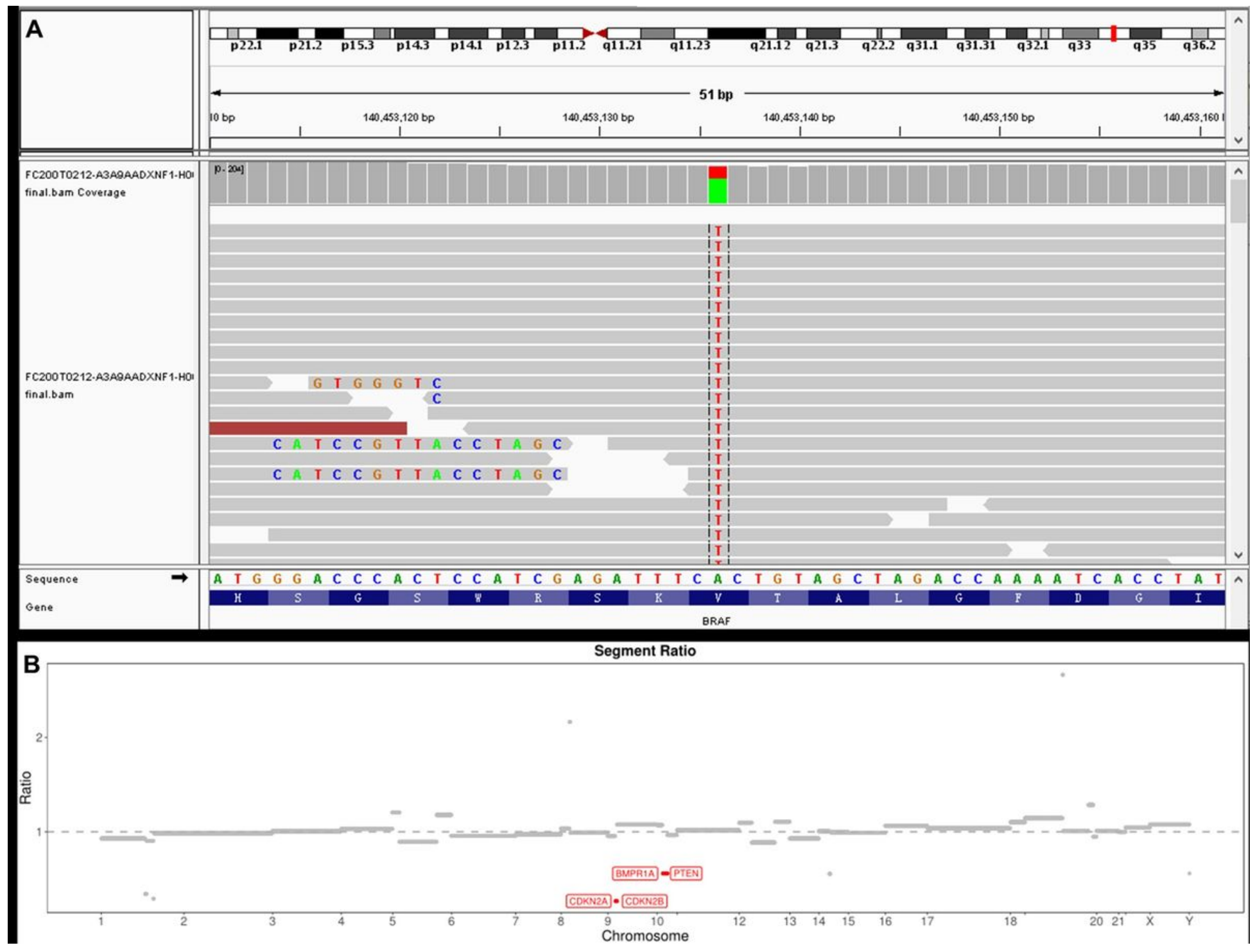

Figure 4

The targeted DNA sequencing (425-cancer-relevant genes) of the first tumor sample. There was a missense_variant mutation (c.1799T>A, p.V600E) in BRAF (A). The deletion of CDKN2A/B, PTEN and BMPR1A were detected (B).

\section{Supplementary Files}

This is a list of supplementary files associated with this preprint. Click to download.

- SupplementaryTable1.docx

- CAREchecklistEnglish.jpg 